\title{
Exact Solutions and Conservation Laws of a (2+1)-Dimensional Nonlinear KP-BBM Equation
}

\author{
Khadijo Rashid Adem and Chaudry Masood Khalique \\ International Institute for Symmetry Analysis and Mathematical Modelling, Department of Mathematical Sciences, \\ North-West University, Mafikeng Campus, Private Bag X 2046, Mmabatho 2735, South Africa
}

Correspondence should be addressed to Chaudry Masood Khalique; masood.khalique@nwu.ac.za

Received 23 January 2013; Accepted 25 March 2013

Academic Editor: Shi Weichen

Copyright ( 2013 K. R. Adem and C. M. Khalique. This is an open access article distributed under the Creative Commons Attribution License, which permits unrestricted use, distribution, and reproduction in any medium, provided the original work is properly cited.

In this paper, we study the two-dimensional nonlinear Kadomtsov-Petviashivilli-Benjamin-Bona-Mahony (KP-BBM) equation. This equation is the Benjamin-Bona-Mahony equation formulated in the KP sense. We first obtain exact solutions of this equation using the Lie group analysis and the simplest equation method. The solutions obtained are solitary waves. In addition, the conservation laws for the KP-BBM equation are constructed by using the multiplier method.

\section{Introduction}

It is well known that the nonlinear evolution equations (NLEEs) are extensively used as models to describe physical phenomena in various fields of sciences, especially in fluid mechanics, solid state physics, plasma physics, plasma waves, and biology. One of the basic physical problems for such models is to obtain their exact solutions. In this respect, during the past few decades, various methods have been developed by researchers to find explicit solutions for the NLEEs. See, for example, Wang et al. [1] and references therein.

The purpose of this paper is to study one such NLEE, namely, the (2+1)-dimensional nonlinear KadomtsovPetviashivilli-Benjamin-Bona-Mahony (KP-BBM) equation that is, given by

$$
\left(u_{t}+u_{x}-\alpha\left(u^{2}\right)_{x}-\beta u_{x x t}\right)_{x}+\gamma u_{y y}=0 .
$$

Here, in (1), $\alpha, \beta$, and $\gamma$ are real valued constants.

The solutions of (1) have been studied in various aspects. See, for example, the recent papers [2-5]. Wazwaz $[2,3]$ used the sine-cosine method, the tanh method and the extended tanh method for finding solitonary solutions of this equation. Abdou [4] used the extended mapping method with symbolic computation to obtain some periodic solutions, solitary wave solution, and triangular wave solution.
In this paper, Lie group analysis [6-9] in conjunction with the simplest equation method [10] is employed to obtain some exact solutions of (1). In addition to this conservation laws will be derived for (1) using the multiplier method [11].

\section{Symmetry Analysis}

In this section, we first calculate the Lie point symmetries of (1) and later use them to construct exact solutions.

2.1. Lie Point Symmetries. A Lie point symmetry of a partial differential equation (PDE) is an invertible transformation of the dependent and independent variables that leaves the equation unchanged. In general, determining all the symmetries of a partial differential equation is a formidable task. However, Sophus Lie observed that if we restrict ourselves to symmetries that depend continuously on a small parameter and that form a group (continuous one-parameter group of transformations), one can linearize the symmetry conditions and end up with an algorithm for calculating continuous symmetries.

The symmetry group of KP-BBM equation (1) will be generated by the vector field of the form

$$
V=\xi^{1} \frac{\partial}{\partial x}+\xi^{2} \frac{\partial}{\partial y}+\xi^{3} \frac{\partial}{\partial t}+\eta \frac{\partial}{\partial u},
$$


where $\xi^{i}, i=1,2,3$ and $\eta$ depend on $x, y, t$, and $u$. Applying the fourth prolongation $\mathrm{pr}^{(4)} V$ to (1), we obtain an overdetermined system of linear partial differential equations. Solving resultant system of linear overdetermined partial differential equations one obtains the following four Lie point symmetries:

$$
\begin{gathered}
V_{1}=\frac{\partial}{\partial x} \\
V_{2}=\frac{\partial}{\partial y} \\
V_{3}=\frac{\partial}{\partial t} \\
V_{4}=-\alpha y \frac{\partial}{\partial y}-2 \alpha t \frac{\partial}{\partial t}+(2 \alpha u-1) \frac{\partial}{\partial u}
\end{gathered}
$$

2.2. Exact Solutions. One of the main reasons for finding symmetries of a differential equation is to use them for finding exact solutions. In this subsection, we will utilize the symmetries calculated in the previous subsection to deduce exact solutions of (1).

One way to obtain exact solutions of (1) is by reducing it to ordinary differential equations. This can be achieved with the use of Lie point symmetries admitted by (1). It is well known that the reduction of a partial differential equation with respect to $r$-dimensional (solvable) subalgebra of its Lie symmetry algebra leads to reducing the number of independent variables by $r$.

First of all, we utilize the symmetry $V=V_{1}+V_{2}+V_{3}$ and reduce the KP-BBM equation (1) to a PDE in two independent variables. It can be seen that the symmetry $V$ yields the following three invariants:

$$
f=t-y, \quad g=x-y, \quad \theta=u .
$$

Now treating $\theta$ as the new dependent variable and $f$ and $g$ as new independent variables, the KP-BBM equation (1) transforms to

$$
\begin{aligned}
\theta_{f g}+ & \theta_{g g}-2 \alpha \theta_{g}^{2}-2 \alpha \theta \theta_{g g}-\beta \theta_{f g g g} \\
& +\gamma \theta_{f f}+2 \gamma \theta_{f g}+\gamma \theta_{g g}=0
\end{aligned}
$$

which is a nonlinear PDE in two independent variables. We now use the Lie point symmetries of (5) and transform it to an ordinary differential equation (ODE). Equation (5) has the following two translational symmetries:

$$
\begin{aligned}
& \Gamma_{1}=\frac{\partial}{\partial g}, \\
& \Gamma_{2}=\frac{\partial}{\partial f} .
\end{aligned}
$$

The combination $\rho \Gamma_{1}+\Gamma_{2}$, where $\rho \neq 0$ is a constant, of the two symmetries $\Gamma_{1}$ and $\Gamma_{2}$ yields the following two invariants:

$$
r=\frac{\rho f-g}{\rho}, \quad \psi=\theta .
$$

This gives rise to a group invariant solution $\psi=\psi(r)$ and consequently using these invariants, (5) is transformed into the fourth-order nonlinear ODE

$$
\begin{aligned}
& -2 \alpha \rho \psi^{\prime 2}-2 \alpha \rho \psi \psi^{\prime \prime} \\
& \quad+\left(\rho+\gamma \rho-\rho^{2}-2 \gamma \rho^{2}+\gamma \rho^{3}\right) \psi^{\prime \prime}+\beta \psi^{\prime \prime \prime \prime}=0 .
\end{aligned}
$$

Integrating the above equation twice and taking the constants of integration to be zero, we obtain a second-order ODE

$$
\rho(-1-\gamma+\gamma \rho)(1-\rho) \psi-\alpha \rho \psi^{2}+\beta \psi^{\prime \prime}=0 .
$$

Multiplying (9) by $\psi^{\prime}$, integrating once and taking the constant of integration to be zero, we obtain the first-order ODE

$$
\frac{1}{2} \rho(-1-\gamma+\gamma \rho)(1-\rho) \psi^{2}-\frac{1}{3} \alpha \rho \psi^{3}+\frac{1}{2} \beta \psi^{\prime 2}=0 .
$$

One can integrate the above equation by separating the variables. After integrating and reverting back to the original variables, we obtain the following group-invariant solutions of the KP-BBM equation (1):

$$
u(x, y, t)=A_{1} \operatorname{sech}^{2}\left[\frac{1}{2}\left( \pm A_{2} r-A_{3} C\right)\right]
$$

where

$$
\begin{gathered}
A_{1}=\frac{1}{2 \alpha^{3}}(-1+\rho)(-1-\gamma+\gamma \rho), \\
A_{2}=\frac{\sqrt{\rho(1-\rho)(-1-\gamma+\gamma \rho)}}{\sqrt{\beta}}, \\
A_{3}=\sqrt{3(-1+\rho)(-1-\gamma+\gamma \rho)}, \\
r=\frac{t \rho-x+(1-\rho) y}{\rho} .
\end{gathered}
$$

To find another group-invariant solution of the KP-BBM equation (1), we now make use of the symmetry $\Gamma=a_{1} V_{1}+$ $a_{2} V_{2}+a_{3} V_{3}+a_{4} V_{4}\left(a_{i}, i=1, \ldots, 4\right.$ are constants $)$. The symmetry $\Gamma$ yields the following three invariants:

$$
\begin{gathered}
f=\frac{2 a_{3} \alpha t-a_{4}}{2 a_{3} \alpha\left(a_{2}-a_{3} \alpha y\right)^{2}}, \quad g=x+\frac{a_{1} \ln \left(a_{2}-a_{3} \alpha y\right)}{a_{3} \alpha}, \\
\theta=a_{2}^{2} u-2 a_{2} a_{3} \alpha y u+a_{3}^{2} \alpha^{2} y^{2} u+a_{2} a_{3} y-\frac{1}{2} a_{3}^{2} \alpha y^{2} .
\end{gathered}
$$

Treating $\theta$ as the new dependent variable and $f$ and $g$ as new independent variables, the KP-BBM equation (1) transforms to a nonlinear fourth-order PDE in two independent variables, namely,

$$
\begin{aligned}
a_{2}^{2} \theta_{g g} & -3 a_{2}^{2} a_{3}^{2} \alpha \gamma+14 a_{3}^{2} \alpha^{2} \gamma f \theta_{f}+a_{1}^{2} \gamma \theta_{g g} \\
& -4 a_{1} a_{3} \alpha \gamma f \theta_{f g}-2 \alpha \theta \theta_{g g} \theta_{f g} \\
& -2 \alpha \theta_{g}^{2}+6 a_{3}^{2} \alpha^{2} \gamma \theta+4 a_{3}^{2} \alpha^{2} \gamma f^{2} \theta_{f f} \\
& -5 a_{1} a_{3} \alpha \gamma \theta_{g}-\beta \theta_{f g g g}=0 .
\end{aligned}
$$


Equation (14) has a single Lie point symmetry, namely,

$$
\Gamma_{1}=\frac{\partial}{\partial g}
$$

and this symmetry yields the two invariants

$$
r=f, \quad \psi=\theta,
$$

which gives a group-invariant solution $\psi=\psi(r)$ and consequently (14) is then transformed to

$$
4 \alpha r^{2} \psi^{\prime \prime}+14 \alpha r \psi^{\prime}+6 \alpha \psi-3 a_{2}^{2}=0,
$$

which is a second-order linear ODE. Now, solving this equation and reverting back to the original variables, we obtain the following solutions of the KP-BBM equation (1):

$$
u(x, y, t)=A_{1}\left(A_{2}+C_{1} r^{-3 / 2}+C_{2} r^{-1}+A_{3}\right),
$$

where $C_{1}$ and $C_{2}$ are constants of integration and

$$
\begin{gathered}
A_{1}=\frac{1}{a_{2}^{2}-2 a_{2} a_{3} \alpha y+a_{3}^{2} \alpha^{2} y^{2}}, \\
A_{2}=\frac{1}{2} a_{3}^{2} \alpha y^{2}-a_{2} a_{3} y, \\
A_{3}=\frac{a_{2}^{2}}{2 \alpha}, \\
r=\frac{2 a_{3} \alpha t-a_{4}}{2 a_{3} \alpha\left(a_{2}-a_{3} \alpha y\right)^{2}} .
\end{gathered}
$$

\section{Simplest Equation Method}

We now use the simplest equation method, which was introduced by Kudryashov [10] and modified by Vitanov [12], to solve the fourth-order ODE (8). The simplest equations that will be used are the Bernoulli and Riccati equations. Their solutions can be written in elementary functions [13].

Let us consider the solution of (8) in the form

$$
\psi(r)=\sum_{i=0}^{M} \mathscr{A}_{i}(H(r))^{i}
$$

where $H(r)$ satisfies the Bernoulli and Riccati equations, $M$ is a positive integer that can be determined by balancing procedure as in [12], and $A_{0}, \ldots, A_{M}$ are parameters to be determined.

3.1. Solutions of (8) Using the Equation of Bernoulli as the Simplest Equation. The balancing procedure [12] yields $M=$ 2 so the solutions of (20) are of the form

$$
\psi(r)=A_{0}+A_{1} H+A_{2} H^{2} .
$$

Substituting (21) into (8) and making use of the Bernoulli equation [13] and then equating all coefficients of the functions $H^{i}$ to zero, we obtain an algebraic system of equations in terms of $A_{0}, A_{1}$, and $A_{2}$.
Solving the system of algebraic equations, we obtain

$$
\begin{gathered}
A_{0}=\frac{a^{2} \beta+\rho+\gamma \rho-\rho^{2}-2 \gamma \rho^{2}+\gamma \rho^{3}}{2 \alpha \rho}, \\
A_{1}=\frac{6 a b \beta}{\alpha}, \\
A_{2}=\frac{6 b^{2} \beta}{\alpha \rho} .
\end{gathered}
$$

Therefore, the solution of (1) is given by

$$
\begin{aligned}
u(x, y, t) & \\
= & A_{0}+A_{1}\left(\frac{\cosh [a(r+C)]+\sinh [a(r+C)]}{1-b \cosh [a(r+C)]-b \sinh [a(r+C)]}\right) \\
& +A_{2}\left(\frac{\cosh [a(r+C)]+\sinh [a(r+C)]}{1-b \cosh [a(r+C)]-b \sinh [a(r+C)]}\right)^{2},
\end{aligned}
$$

where $r=t \rho-x+(1-\rho) y / \rho$, and $C$ is a constant of integration.

3.2. Solutions of (8) Using Riccati Equation as the Simplest Equation. The balancing procedure yields $M=2$, so the solutions of (20) are of the form

$$
\psi(r)=A_{0}+A_{1} H+A_{2} H^{2} .
$$

Substituting (24) into (8) and making use of the Riccati equation [13], we obtain algebraic equations in terms of $A_{0}$, $A_{1}$, and $A_{2}$ by equating all coefficients of the functions $H^{i}$ to zero.

Solving the algebraic equations, one obtains

$$
\begin{gathered}
A_{0}=\frac{b^{2} \beta+\rho+\gamma \rho-\rho^{2}-2 \gamma \rho^{2}+\gamma \rho^{3}}{2 \alpha \rho}, \\
A_{1}=0 \\
A_{2}=\frac{2\left(2 a^{3} \beta+11 a^{2} b \beta+2 a b^{2} \beta\right)}{\alpha \rho(2 a+3 b)},
\end{gathered}
$$

and hence the solutions of (1) are

$$
\begin{aligned}
& u(x, y, t)=A_{0}+A_{2}\left(-\frac{b}{2 a}-\frac{\theta}{2 a} \tanh \left[\frac{1}{2} \theta(r+C)\right]\right)^{2}, \\
& u(x, y, t) \\
& =A_{0}+A_{2}\left(-\frac{b}{2 a}-\frac{\theta}{2 a} \tanh \left(\frac{1}{2} \theta r\right)\right. \\
& \left.+\frac{\operatorname{sech}(\theta r / 2)}{C \cosh (\theta r / 2)-(2 a / \theta) \sinh (\theta r / 2)}\right)^{2},
\end{aligned}
$$

where $r=t \rho-x+(1-\rho) y / \rho$, and $C$ is a constant of integration. 


\section{Conservation Laws}

In this section, we construct conservation laws for (1). The multiplier method will be used [11]. See also [14].

Consider a $k$ th-order system of PDEs of $n$ independent variables $x=\left(x^{1}, x^{2}, \ldots, x^{n}\right)$ and $m$ dependent variables $u=$ $\left(u^{1}, u^{2}, \ldots, u^{m}\right)$, namely,

$$
E_{\alpha}\left(x, u, u_{(1)}, \ldots, u_{(k)}\right)=0, \quad \alpha=1, \ldots, m,
$$

where $u_{(1)}, u_{(2)}, \ldots, u_{(k)}$ denote the collections of all first, second, ..., $k$ th-order partial derivatives, that is, $u_{i}^{\alpha}=D_{i}\left(u^{\alpha}\right)$, $u_{i j}^{\alpha}=D_{j} D_{i}\left(u^{\alpha}\right), \ldots$, respectively, with the total derivative operator with respect to $x^{i}$ is given by

$$
D_{i}=\frac{\partial}{\partial x^{i}}+u_{i}^{\alpha} \frac{\partial}{\partial u^{\alpha}}+u_{i j}^{\alpha} \frac{\partial}{\partial u_{j}^{\alpha}}+\cdots, \quad i=1, \ldots, n,
$$

where the summation convention is used whenever appropriate [9].

The following are known (see, e.g., [9] and the references therein).

The Euler-Lagrange operator, for each $\alpha$, is given by

$$
\frac{\delta}{\delta u^{\alpha}}=\frac{\partial}{\partial u^{\alpha}}+\sum_{s \geq 1}(-1)^{s} D_{i_{1}} \cdots D_{i_{s}} \frac{\partial}{\partial u_{i_{1} i_{2} \cdots i_{s}}^{\alpha}}, \quad \alpha=1, \ldots, m \text {. }
$$

The $n$-tuple vector $T=\left(T^{1}, T^{2}, \ldots, T^{n}\right), T^{j} \in \mathscr{A}, j=1, \ldots, n$, is a conserved vector of (27) if $T^{i}$ satisfies

$$
\left.D_{i} T^{i}\right|_{(27)}=0
$$

Equation (30) defines a local conservation law of system (27).

A multiplier $\Lambda_{\alpha}\left(x, u, u_{(1)}, \ldots\right)$ has the property that

$$
\Lambda_{\alpha} E_{\alpha}=D_{i} T^{i}
$$

holds identically. Here, we will consider multipliers of the second order, that is, $\Lambda_{\alpha}=\Lambda_{\alpha}\left(t, x, u, v, w, u_{x}, v_{x}\right.$, $\left.w_{x}, u_{x x}, v_{x x}, w_{x x}\right)$. The right hand side of (31) is a divergence expression. The determining equation for the multiplier $\Lambda_{\alpha}$ is

$$
\frac{\delta\left(\Lambda_{\alpha} E_{\alpha}\right)}{\delta u^{\alpha}}=0
$$

Once the multipliers are obtained, the conserved vectors are calculated via a homotopy formula [11].

4.1. Construction of Conservation Laws for KP-BBM Equation. We now construct conservation laws for the $(2+1)$ dimensional nonlinear KP-BBM equation (1). The zerothorder multiplier for $(1)$ is, $\Lambda(t, x, y, u)$, that is, given by

$$
\Lambda=C_{1} x+C_{1} t x-\frac{C_{1} y^{2}}{2 \gamma}+y F_{2}(t)+F_{3}(t),
$$

where $C_{1}$ is a constant, and $F_{2}(t)$ and $F_{3}(t)$ are arbitrary functions of $t$. Corresponding to the above multiplier, we have the following conserved vectors of (33):

$$
\begin{aligned}
T_{1}^{t}=\frac{1}{8 \gamma}\{ & -4 \gamma t u-4 \gamma u+2 \beta \gamma t u_{x x}-2 \beta \gamma t x u_{x x x}+4 \gamma t x u_{x} \\
& +2 \beta \gamma u_{x x}-2 \beta \gamma x u_{x x x} \\
& \left.+4 \gamma x u_{x}+\beta y^{2} u_{x x x}-2 y^{2} u_{x}\right\} \\
T_{1}^{x}=\frac{1}{8 \gamma}\{ & \left\{\alpha y^{2} u_{x} u-16 \alpha \gamma t x u_{x} u-16 \alpha \gamma x u_{x} u\right.
\end{aligned}
$$

Remark 1. Due to the presence of the arbitrary function in the multiplier, one can obtain an infinitely many conservation laws for the (2+1)-dimensional nonlinear KP-BBM equation.

\section{Concluding Remarks}

In this paper, we obtained the solutions of the twodimensional nonlinear Kadomtsov-Petviashivilli-BenjaminBona-Mahony (KP-BBM) equation by employing the Lie group analysis and the simplest equation method. The solutions obtained are solitary waves and nontopological solutions. The conservation laws for the underlying equation were also derived by using the multiplier method. 


\section{References}

[1] M. Wang, X. Li, and J. Zhang, “The $\left(G^{\prime} / G\right)$-expansion method and travelling wave solutions of nonlinear evolution equations in mathematical physics," Physics Letters A, vol. 372, no. 4, pp. 417-423, 2008.

[2] A. M. Wazwaz, "Exact solutions of compact and noncompact structures for the KP-BBM equation," Applied Mathematics and Computation, vol. 169, no. 1, pp. 700-712, 2005.

[3] A. M. Wazwaz, "The extended tanh method for new compact and noncompact solutions for the KP-BBM and the ZK-BBM equations," Chaos, Solitons and Fractals, vol. 38, no. 5, pp. 15051516, 2008.

[4] M. A. Abdou, "Exact periodic wave solutions to some nonlinear evolution equations," International Journal of Nonlinear Science, vol. 6, no. 2, pp. 145-153, 2008.

[5] M. Song, C. Yang, and B. Zhang, "Exact solitary wave solutions of the Kadomtsov-Petviashvili-Benjamin-Bona-Mahony equation," Applied Mathematics and Computation, vol. 217, no. 4, pp. 1334-1339, 2010.

[6] G. W. Bluman and S. Kumei, Symmetries and Differential Equations, vol. 81 of Applied Mathematical Sciences, Springer, New York, NY, USA, 1989.

[7] P. J. Olver, Applications of Lie Groups to Differential Equations, vol. 107 of Graduate Texts in Mathematics, Springer, Berlin, Germany, 2nd edition, 1993.

[8] L. V. Ovsiannikov, Group Analysis of Differential Equations, Academic Press, New York, NY, USA, 1982.

[9] N. H. Ibragimov, CRC Handbook of Lie Group Analysis of Differential Equations, vol. 1-3, CRC Press, Boca Raton, Fla, USA, 1994-1996.

[10] N. A. Kudryashov, "Simplest equation method to look for exact solutions of nonlinear differential equations," Chaos, Solitons and Fractals, vol. 24, no. 5, pp. 1217-1231, 2005.

[11] S. C. Anco and G. Bluman, "Direct construction method for conservation laws of partial differential equations-I: examples of conservation law classifications," European Journal of Applied Mathematics, vol. 13, no. 5, pp. 545-566, 2002.

[12] N. K. Vitanov, "Application of simplest equations of Bernoulli and Riccati kind for obtaining exact traveling-wave solutions for a class of PDEs with polynomial nonlinearity," Communications in Nonlinear Science and Numerical Simulation, vol. 15, no. 8, pp. 2050-2060, 2010.

[13] A. R. Adem and C. M. Khalique, "Symmetry reductions, exact solutions and conservation laws of a new coupled $\mathrm{KdV}$ system," Communications in Nonlinear Science and Numerical Simulation, vol. 17, no. 9, pp. 3465-3475, 2012.

[14] M. Anthonyrajah and D. P. Mason, "Conservation laws and invariant solutions in the Fanno model for turbulent compressible flow," Mathematical \& Computational Applications, vol. 15, no. 4, pp. 529-542, 2010. 


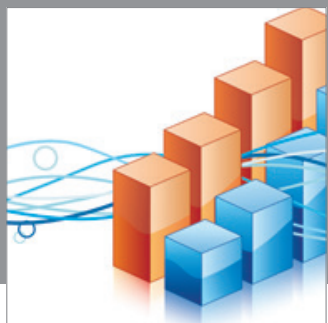

Advances in

Operations Research

mansans

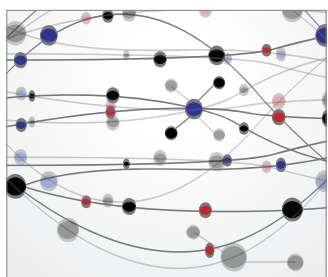

The Scientific World Journal
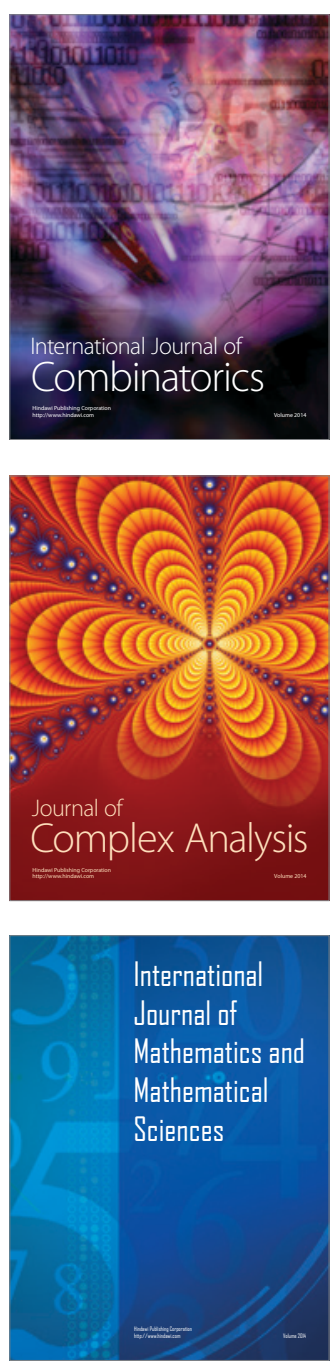
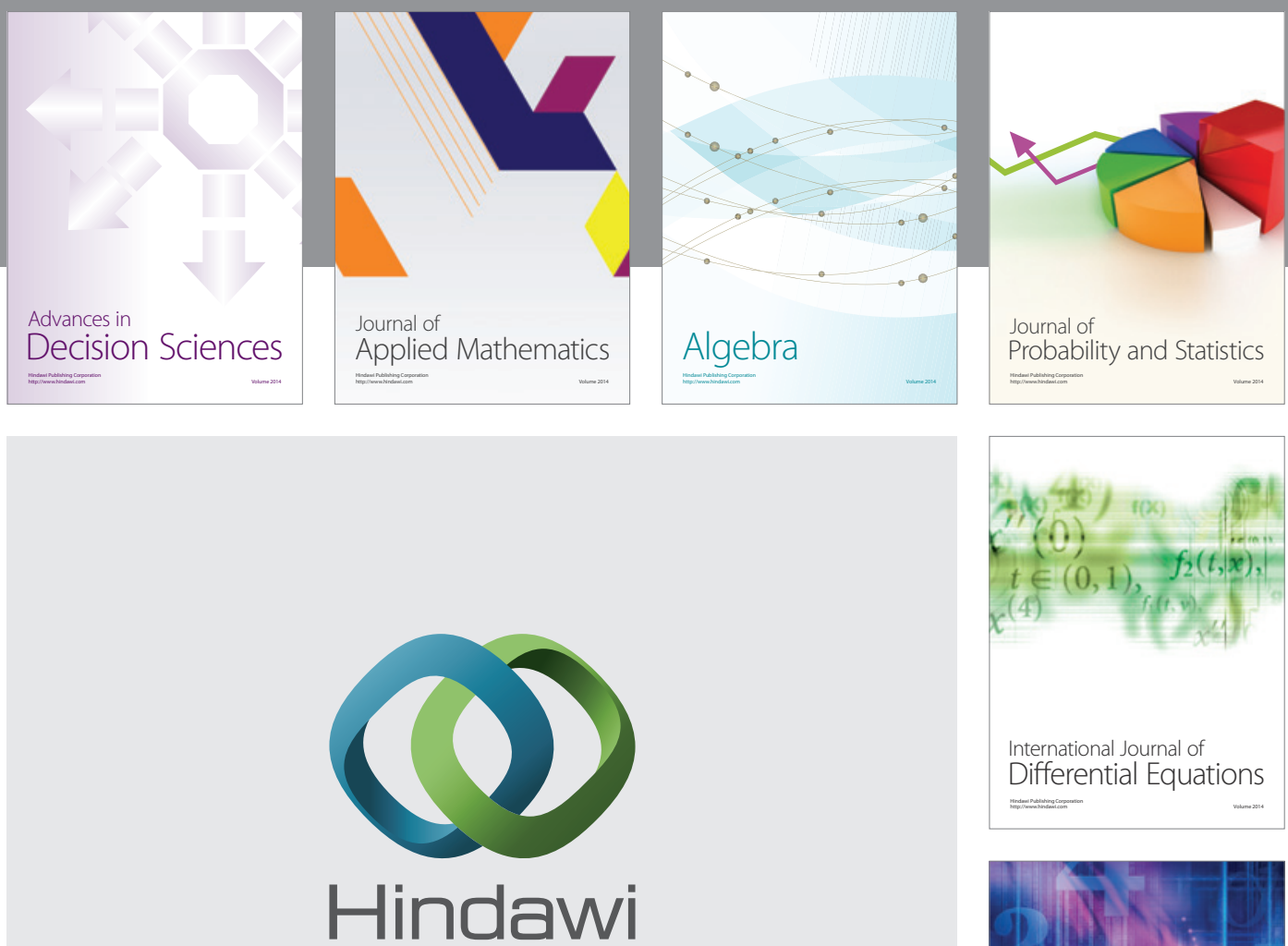

Submit your manuscripts at http://www.hindawi.com
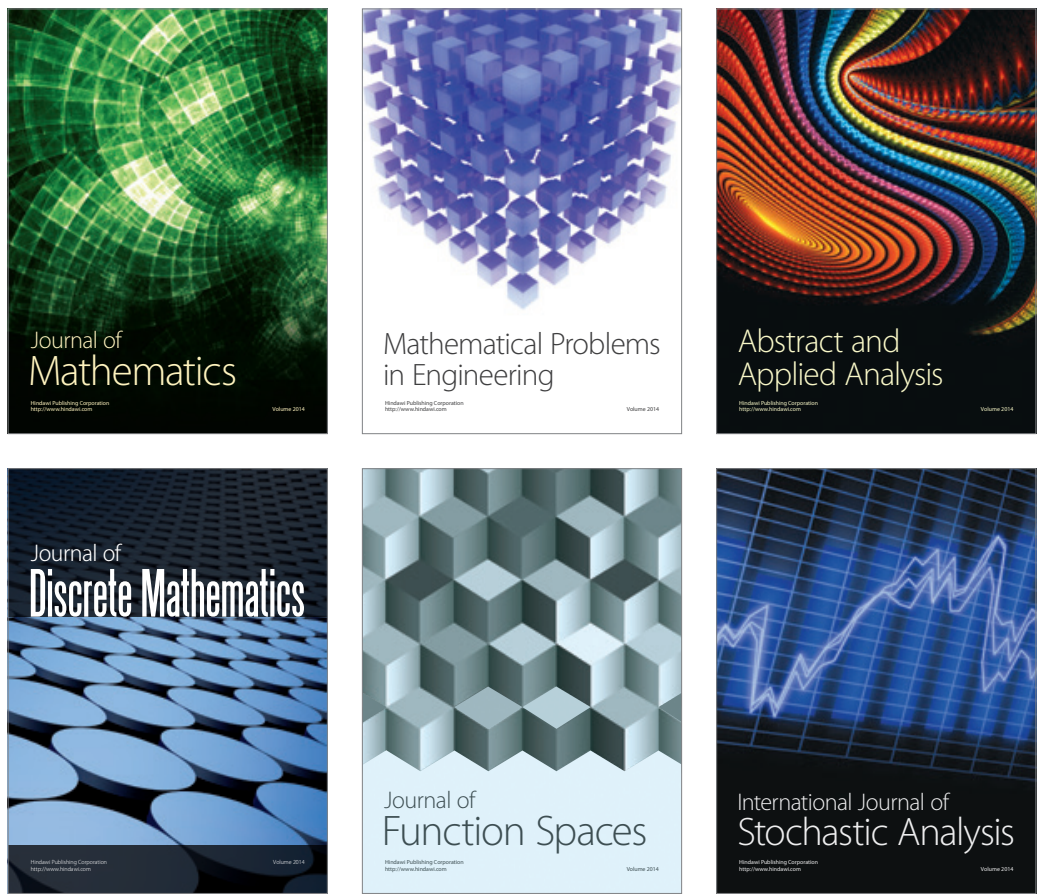

Journal of

Function Spaces

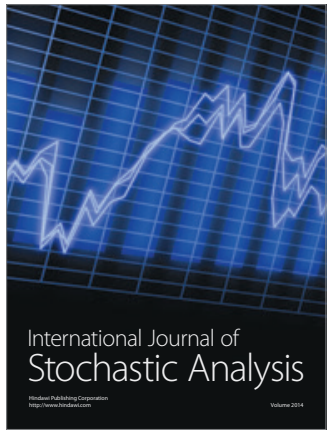

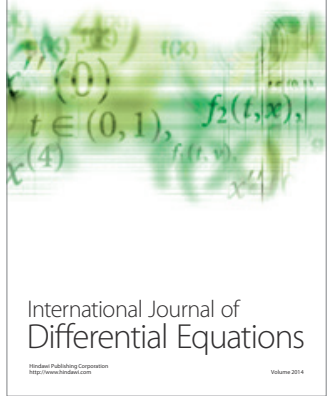
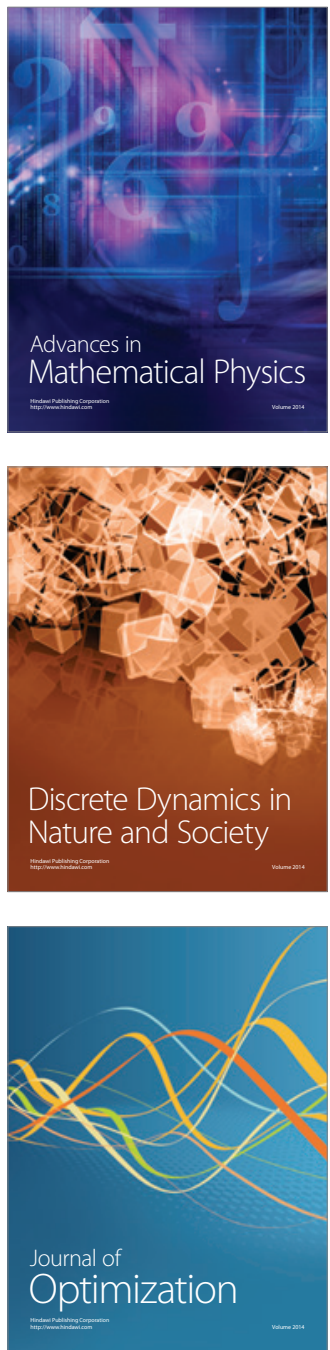